\title{
BMJ Open Prospective association between social engagement and cognitive impairment among middle-aged and older adults: evidence from the China Health and Retirement Longitudinal Study
}

\author{
Shuduo Zhou (D) , ${ }^{1,2}$ Suhang Song, ${ }^{3}$ Yinzi Jin (D) , ${ }^{1,2}$ Zhi-Jie Zheng ${ }^{1,2}$
}

To cite: Zhou S, Song S, Jin Y, et al. Prospective association between social engagement and cognitive impairment among middle-aged and older adults: evidence from the China Health and Retirement Longitudinal Study. BMJ Open 2020;10:e040936. doi:10.1136/ bmjopen-2020-040936

- Prepublication history and additional materials for this paper is available online. To view these files, please visit the journal online (http://dx.doi. org/10.1136/bmjopen-2020040936).

Received 26 May 2020 Revised 18 0ctober 2020 Accepted 26 0ctober 2020

Check for updates

(C) Author(s) (or their employer(s)) 2020. Re-use permitted under CC BY-NC. No commercial re-use. See rights and permissions. Published by BMJ.

${ }^{1}$ Department of Global Health, Peking University Health Science Centre, Beijing, China

${ }^{2}$ Institute for Global Health,

Peking University, Beijing, China

${ }^{3}$ China Center for Health

Development Studies, Peking

University, Beijing, China

Correspondence to

Dr Yinzi Jin; yzjin@bjmu.edu.cn

\section{ABSTRACT}

Objectives Cognitive impairment is a precursor of dementia. This study aimed to examine the association of social engagement with cognitive decline during the process of normal ageing.

Methods We used data from the China Health and Retirement Longitudinal Study. The cognitive functions were tested at baseline, and 2-year and 4-year follow-up visits. Social engagement was constructed as a comprehensive measure including the quantity and frequency of social activities. Activities of social engagement were classified into three types. Multilevel logistic model was fitted to evaluate the prospective association between social engagement and cognitive impairment.

Results After 2-year follow-up, compared with participants with the lowest level of social engagement, those with level-2, level-3 and level-4 social engagement had a $12 \%(0 \mathrm{R}=0.88,95 \% \mathrm{Cl} 0.71$ to $1.09, \mathrm{p}=0.242)$, $17 \%(\mathrm{OR}=0.83,95 \% \mathrm{Cl} 0.71$ to $0.97, \mathrm{p}=0.020)$ and $25 \%$ $(\mathrm{OR}=0.75,95 \% \mathrm{Cl} 0.61$ to $0.93, \mathrm{p}=0.008)$ lower risk for developing cognitive impairment, respectively. A similar pattern of significantly protective association was found at 4-year follow-up. Combined effect analysis showed that participants who attended one type of social engagement had a lower risk of developing cognitive impairment and the protective effect was even larger with those who attended two or three types of social engagement. Conclusions Social engagement had a protective effect on cognitive impairment among middle-aged and older adults in China. Given the growing epidemic of dementia and rapid pace of ageing in low-income and middleincome countries, our study shed lights on comprehensive and tailored public health programmes for improving social engagement, to delay cognitive impairment at mid-age and later life.

\section{INTRODUCTION}

In most cases, the pathological cognitive impairment process may serve as a precursor of dementia, ${ }^{12}$ which is diagnosed by severe neurocognitive disorder with social or occupational disfunction. In
Strengths and limitations of this study

- This is the first nationally representative study that investigated the prospective association between social engagement and cognitive impairment in lowincome and middle-income countries.

- Social engagement had a significant protective effect on the cognitive impairment among individuals aged 45 years and over.

- Relative to single type of social engagement, multiple types of social engagement showed a greater protective effect on cognitive impairment.

- Comprehensive and tailored programmes for improving the enrolment of social engagement are imperative to slow down cognitive decline.

- The observational nature of our study limited our ability to confirm a causal relationship between social engagement and cognitive impairment.

the population-ageing society, dementia has been a serious public health problem with the number doubling during the past two decades. ${ }^{3}$ Cognitive impairment, which often begins at age around 45 , is a pathophysiological process of brain changes associated with age. ${ }^{45}$ Delaying the process of cognitive decline could prevent dementia at later life. Nearly $20 \%$ of Chinese adults aged 60 or above had mild cognitive impairment, of which $6 \%$ developed dementia. ${ }^{6}$ People with cognitive impairment have difficulty in performing daily living tasks and need more medical and family care, thus leads to a substantial economic and social impact. ${ }^{78}$ The burden of cognitive impairment is increasing on ageing populations in China. ${ }^{9}$ Therefore, it is necessary to identify potential influencing factors that could prevent individuals from cognitive impairment, such information is needed for designing public health interventions. ${ }^{10}$ 
Prior studies suggest that individuals who are more socially engaged tend to have better cognitive functions compared with the less engaged. ${ }^{11}{ }^{12}$ In general, social engagement refers to maintenance of social connections and participation in social activities. ${ }^{11}{ }^{13}$ Different hypotheses have been used to explain potential mechanisms of social engagement on cognitive impairment, including biological, behavioural and psychological aspects. Cognitive reserve is one of the most commonly referred hypotheses. It posits education or life experience influence neural processing and synaptic organisation, and contribute to increased reserve, thus allowing one to cope with cognitive decline better than others. ${ }^{14} 15$ Social engagement includes participating in social activities, interacting with social members and sharing resources of education, culture, religion and politics that in turn enhance cognitive reserve. ${ }^{16}$ On the other hand, social engagement may also lead to more positive emotional status and lower stress, better diet and healthier lifestyle, which promote physiological and psychological wellbeing and better cognitive function. ${ }^{1718}$

A few studies have examined the cross-sectional association between cognitive impairment and social engagement, the causal relationship, however, has been established. It is not clear whether social engagement leads to better cognitive performance, or rather that individuals with better cognitive function tend to participate more in social engagement. ${ }^{151920}$ In addition, to our knowledge, few studies have explored the combined effect of multiple activities of social engagement on cognitive impairment giving the diverse activities might facilitate different cognitive functioning. However, activities of social engagement showed heterogeneity in prior studies. One key question remained unanswered: does the effect on cognitive function found in other settings apply to specific daily activities in Chinese culture, where the progress of ageing and urbanisation is unprecedented rapid and social activities change quickly. Furthermore, cognitive impairment appears to be more pronounced in lowand middle-income countries (LMICs), which further contribute to population burden of disease. ${ }^{21} 22$ Despite this, evidence on the potential protective association between social engagement and cognitive impairment in LMICs remains relatively scarce.

To fill this gap, this study aims to (1) assess the prospective association between social engagement and cognitive impairment among middle-aged and older people in China and (2) investigate single and the combined effects of different types of social engagement on cognitive impairment over time.

\section{METHOD}

\section{Study design, setting and population}

The China Health and Retirement Longitudinal Study (CHARLS) is a biennial prospective cohort study of nationally representative of Chinese adults aged $\geq 45$ years and their spouses. CHARLS used multistage probability

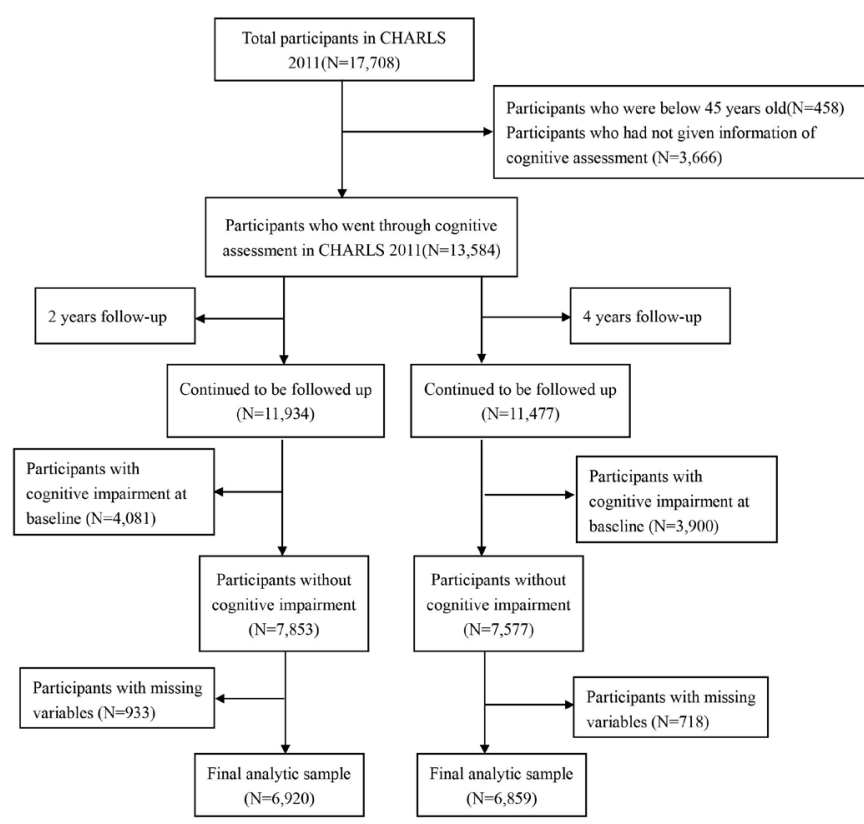

Figure 1 Flowchart of the study sample. CHARLS, China Health and Retirement Longitudinal Study.

sampling to select 150 county-level units in which 450 communities chosen within 28 provinces of China. ${ }^{23}$ The survey included three phases: the baseline survey (20112012) with 17708 participants recruited and enrolled, the 2-year follow-up survey (2013-2014) with 89\% reinterviewed and the 4-year follow-up survey (2015-2016) with $73 \%$ finally reinterviewed.

We used data from 2 years and 4 years of follow-up visits (2011-2012, 2013-2014 and 2015-2016). Of the total 17708 participants at baseline survey (2011-2012), 458 participants aged $<45$ years old and 3666 did not complete the social engagement measures or cognitive testing, thus a total of 13584 participants had cognitive assessment at the baseline. We excluded individuals who had dementia or cognitive impairment without dementia (CIND) at baseline $(\mathrm{n}=4081)$, lost to follow-up at 2-year survey $(n=1650)$, with missing data in critical variables $(n=928)$. For 4 years of follow-up visits, we excluded individuals who had dementia or CIND at baseline $(n=3900)$, lost to follow-up at 4-year survey $(\mathrm{n}=2107)$, or missing data in critical variables $(n=713)$. Figure 1 presented a flow chart for inclusion and exclusions of the study sample from the 2011-2012 CHARLS through the follow-up surveys.

\section{Measurements}

\section{Cognitive impairment}

In the CHARLS Survey, cognitive function was measured through a global cognitive function score based on two aspects of cognitive functions: episodic memory and mental intactness. ${ }^{24}{ }^{25}$ For episodic memory, each participant was asked to repeat as many as possible 10 Chinese nouns just read to him/her (immediate word recall), and then to recall the same 10-word list 5 min later (delayed recall). Answers to these questions were aggregated into a single episodic memory score ranging from 0 to 20 . 
Table 1 Two-year and four-year incidence of cognitive impairment among participants free of cognitive impairment at baseline: The China Health and Retirement Longitudinal Study

\begin{tabular}{|c|c|c|c|c|c|c|}
\hline \multirow[b]{2}{*}{ Variables } & \multicolumn{3}{|c|}{ Two-year follow-up } & \multicolumn{3}{|c|}{ Four-year follow-up } \\
\hline & $\mathbf{N}$ & Cognitive impairment & $P$ value & $\mathbf{N}$ & Cognitive impairment & $P$ value \\
\hline Total, n (\%) & 6920 & $1290(18.6)$ & & 6859 & $1574(23.0)$ & \\
\hline Age, n (\%) & & & $<0.001$ & & & $<0.001$ \\
\hline$<60$ & 4394 & $675(15.4)$ & & 4442 & $816(18.4)$ & \\
\hline $60-69$ & 1945 & $411(21.1)$ & & 1914 & $541(28.3)$ & \\
\hline $70-79$ & 527 & $180(34.2)$ & & 463 & $193(41.7)$ & \\
\hline 80 or above & 54 & $24(44.4)$ & & 40 & $24(60.0)$ & \\
\hline Sex, n (\%) & & & $<0.001$ & & & 0.005 \\
\hline Female & 3165 & $667(21.1)$ & & 3131 & 767 (24.5) & \\
\hline Male & 3755 & $623(16.6)$ & & 3728 & $807(21.7)$ & \\
\hline Education, n (\%) & & & $<0.001$ & & & $<0.001$ \\
\hline Illiterate & 798 & $378(47.4)$ & & 806 & $434(53.8)$ & \\
\hline Part of primary school & 1143 & $325(28.4)$ & & 1136 & $402(35.4)$ & \\
\hline Primary school & 1764 & $342(19.4)$ & & 1744 & $440(25.2)$ & \\
\hline Middle school & 1981 & $192(9.7)$ & & 1996 & $235(11.8)$ & \\
\hline High school or above & 1234 & $53(4.3)$ & & 1177 & $63(5.4)$ & \\
\hline Marriage, $\mathrm{n}(\%)$ & & & $<0.001$ & & & $<0.001$ \\
\hline Having spouse & 6387 & 1145 (17.9) & & 6341 & $1402(22.1)$ & \\
\hline No spouse & 533 & $145(27.2)$ & & 518 & 172 (33.2) & \\
\hline Residence, n (\%) & & & $<0.001$ & & & $<0.001$ \\
\hline Rural & 3874 & $894(23.1)$ & & 3947 & 1089 (27.6) & \\
\hline Urban & 3046 & $396(13.0)$ & & 2912 & $485(16.7)$ & \\
\hline Smoke, n (\%) & & & 0.052 & & & 0.451 \\
\hline Current & 2312 & $444(19.2)$ & & 2333 & $554(23.8)$ & \\
\hline Former & 683 & $104(15.2)$ & & 655 & $142(21.7)$ & \\
\hline Never & 3925 & $742(18.9)$ & & 3871 & $878(22.7)$ & \\
\hline Alcohol drinker, n (\%) & & & $<0.001$ & & & $<0.001$ \\
\hline Current & 2536 & 395 (15.6) & & 2527 & $515(20.4)$ & \\
\hline Former & 544 & $103(18.9)$ & & 540 & $145(26.9)$ & \\
\hline Never & 3840 & $792(20.6)$ & & 3792 & $914(24.1)$ & \\
\hline Hypertension, n (\%) & & & 0.106 & & & 0.215 \\
\hline Yes & 1792 & 357 (19.9) & & 1731 & $416(24.0)$ & \\
\hline No & 5128 & 933 (18.2) & & 5128 & $1158(22.6)$ & \\
\hline Diabetes, n (\%) & & & 0.478 & & & 0.733 \\
\hline Yes & 482 & $84(17.4)$ & & 462 & 109 (23.6) & \\
\hline No & 6438 & $1206(18.7)$ & & 6397 & $1465(22.9)$ & \\
\hline Coronary heart diseases, n (\%) & & & 0.037 & & & 0.027 \\
\hline Yes & 894 & $144(16.1)$ & & 870 & $174(20.0)$ & \\
\hline No & 6026 & $1146(19.0)$ & & 5989 & $1400(23.4)$ & \\
\hline Stroke, n (\%) & & & 0.008 & & & 0.059 \\
\hline Yes & 166 & $44(26.4)$ & & 146 & $43(29.5)$ & \\
\hline No & 6754 & $1246(18.4)$ & & 6713 & $1531(22.8)$ & \\
\hline Depressive symptoms, n (\%) & & & $<0.001$ & & & $<0.001$ \\
\hline Yes & 1999 & $499(25.0)$ & & 1992 & $599(30.1)$ & \\
\hline
\end{tabular}


Table 1 Continued

\begin{tabular}{|c|c|c|c|c|c|c|}
\hline \multirow[b]{2}{*}{ Variables } & \multicolumn{3}{|c|}{ Two-year follow-up } & \multicolumn{3}{|c|}{ Four-year follow-up } \\
\hline & $\mathbf{N}$ & Cognitive impairment & $P$ value & $\mathbf{N}$ & Cognitive impairment & $P$ value \\
\hline No & 4921 & $791(16.1)$ & & 4867 & $975(20.0)$ & \\
\hline ADL score, n (\%) & & & $<0.001$ & & & $<0.001$ \\
\hline 0 & 6183 & 1107 (17.9) & & 6136 & $1366(22.3)$ & \\
\hline $1 \sim 2$ & 601 & $149(24.8)$ & & 591 & $162(27.4)$ & \\
\hline $3 \sim 6$ & 136 & $34(25.0)$ & & 132 & 46 (34.9) & \\
\hline Number of children, $\mathrm{n}(\%)$ & & & $<0.001$ & & & $<0.001$ \\
\hline 0 & 19 & $3(15.8)$ & & 18 & $3(16.7)$ & \\
\hline $1-2$ & 4096 & $597(14.6)$ & & 4079 & $727(17.8)$ & \\
\hline 3 or above & 2805 & $690(24.6)$ & & 2762 & $844(30.6)$ & \\
\hline Weekly contact with children & & & 0.001 & & & 0.046 \\
\hline Yes & 6482 & $1183(18.3)$ & & 6435 & $1460(22.7)$ & \\
\hline No & 438 & 107 (24.4) & & 424 & 114 (26.9) & \\
\hline
\end{tabular}

ADL, activity of daily living.

For mental intactness, measures consisting of numerical ability, time orientation and picture drawing were used to formulate the score. The questions included: serial 7 subtractions from 100 (up to five times), naming the date of the investigation day (month, day, year and date of week), and redrawing a picture of two overlapping pentagons shown to $\mathrm{him} / \mathrm{her}$. Answers to these questions were aggregated into a single mental intactness score ranging from 0 to 10. The score of global cognitive function was the summation of episodic memory and mental intactness scores, ranging from 0 to 30 with a higher score indicating a better cognitive function. The cognitive tests used in CHARLS accorded with the Health Retirement Study and were adapted from the Telephone Interview for Cognitive Status, which provides a valid alternate to rate global cognitive function compared with the MiniMental State Examination. ${ }^{26}$ In addition, previous study had confirmed that the global cognitive function score demonstrated satisfactory validity and reliability among Chinese middle aged and older adults. ${ }^{24} 25$ According to the previous studies, respondent who scored from 0 to 6 were classified as a person with dementia, and 7 to 11 were classified as CIND. ${ }^{27}{ }^{28}$ In the present study, a score of 11 or less was used as a cut point for having cognitive impairment.

\section{Social engagement}

To measure social engagement, participants were asked whether they had done any sorts of the following activities in the last month, which was a multiple-choice question including 10 activities. If the answer was "yes', they were further asked about the frequency of activity. The activities surveyed include: (1) interactions with friends; (2) playing Mahjong, chess, cards or going to community club; (3) helping family, friends or neighbours who do not live with participants; (4) going to sport, social or other kind of club; (5) taking part in a community organisation; (6) voluntary or charity work; (7) caring for a sick or disabled adult who does not live with the respondents and who did not pay for the help; (8) attending educational or training course; (9) stock investment and (10) using internet. As for the frequency of each activity, four choices were given to the respondents, 'almost daily', 'almost every week', 'not regularly' and 'none' in the last month, measured by scores ' 3 ,' ' 2 ,' ' 1 ' and ' 0 ,' respectively.

A composite index of social engagement was constructed by summing the scores for all ten indicators, ranging from 0 to 18 with higher scores indicating better social engagement. Considering the distribution of scores, a four-point categorical rank variable was used to represent the scores of social engagement with score 0 classified into level 1, score 1 classified into level 2, score 2 or 3 classified into level 3 and score 4 or above classified into level 4, higher rank indicating higher scores of social engagement. In addition, based on the type of social engagement, activities surveyed were classified into three categories: leisure activities, social activities helping others and learning activities for new knowledge. ${ }^{29}$ In the 10 activities mentioned above, activities 1, 2, 4 and 5 were defined as leisure activities, 3, 6 and 7 as social activities helping others and 8,9 and 10 as learning activities for new knowledge. To explore the single and combined effect of social engagement on cognitive impairment, our final analytic sample were classified into three categories, including those with none of social engagement, those with one type of social engagement and those with two or more types of social engagement.

\section{Measurements of other related variables}

The International Classification of Function, Disability and Health ${ }^{30}$ outlines that cognitive function is influenced 
by personal factors, environmental factors, health condition and body functions. ${ }^{152}$

The personal factors included age, gender (male or female), educational attainment (illiterate, part of primary school, primary school, middle school, high school or above), marital status (married and currently living with spouse or others), residence status (currently living in rural or urban areas), smoking status (current, former or never) and alcohol drinker (current, former or never). The health condition covariates included whether they had ever been diagnosed with hypertension, diabetes, coronary heart disease, stroke and self-reported depressive symptoms. For the body functions factors, a six-item summary in CHARLS that includes bathing, dressing, eating, getting in/out of bed, using the toilet and controlling urination was selected. The environmental factors mainly referred to family support in this study, including the number of children the respondents had, and whether he/she had a contact weekly with the children in person or by phone.

\section{Statistical analysis}

Descriptive statistical analysis and the Pearson $\chi^{2}$ test were used to analyse the difference in population characteristics between the cognitive impairment and non-cognitive impairment groups. Two pairs of multivariate logistic regression models were used to analyse the prospective association between the social engagement at baseline and incidence of cognitive impairment at 2-year and 4-year follow-up. The first pair of models focused on the social engagement as an independent predictor, and the second pair of models focused on the combined effect of different types of social engagement. All the models were adjusted for the covariates. To evaluate the potential bias due to non-response and attrition across difference survey waves, sensitivity analysis was conducted using the same models with the complete sample who participated in all three waves. The software Stata V.14.1 for Mac (Stata Corp, College Station, Texas, USA) was used for statistical analysis.

\section{Patient and public involvement}

Patients were not involved in the design or management of this research. We used data from the CHARLS with no direct involvement of participants in the design or conduct of the original cohort study.

\section{RESULTS}

\section{Population characteristics}

Table 1 describes the 2-year and 4-year incidence of cognitive impairment among the CHARLS participants and their subgroups who were free of cognitive impairment at baseline. Among 6920 middle-aged and older participants who were followed up for 2years, 1290 (18.6\%) developed cognitive impairment at 2-year follow-up. Among 6859 participants who went through 4-year follow-up, $1574(23.0 \%)$ developed cognitive impairment at 4-year follow-up. The incidence of cognitive impairment was statistically significantly different among subgroups at 2-year and 4-year follow-up. For example, the incidence was higher in women (21.1\% at 2-year follow-up, $24.5 \%$ at 4-year follow-up) than in men $(16.6 \%, 21.7 \%)$, those who lived in rural $(23.1 \%, 27.6 \%)$ than in urban $(13.0 \%$, $16.7 \%)$, those who lived without spouse $(27.2 \%, 33.2 \%)$ than with spouse $(17.9 \%, 22.1 \%)$ and those with depressive symptoms $(25.0 \%, 30.1 \%)$ than without depressive symptoms $(16.1 \%, 20.0 \%)$ (table 1$)$.

\section{Prospective association between social engagement and cognitive impairment}

Table 2 shows the results of the first pair of multivariate logistic regression models. At 2-year follow-up, compared with participants with level-1 (the lowest level) social engagement, participants with level-2, level-3, and level-4 social engagement had a $12 \%(\mathrm{OR}=0.88,95 \% \mathrm{CI}$ 0.71 to $1.09, \mathrm{p}=0.242), 17 \% \quad(\mathrm{OR}=0.83,95 \% \mathrm{CI} 0.71$ to $0.97, \mathrm{p}=0.020)$ and $25 \%(\mathrm{OR}=0.75,95 \% \mathrm{CI} 0.61$ to 0.93 , $\mathrm{p}=0.008$ ) lower risk for developing cognitive impairment, respectively. A similar pattern of significantly protective association between social engagement and cognitive impairment was found at 4-year follow-up. For example, compared with the participants with level-1 social engagement, the participants with level- 4 social engagement had a $27 \%$ (OR=0.73, 95\% CI 0.60 to $0.89, \mathrm{p}=0.002)$ lower risk for developing cognitive impairment at 4-year follow-up (table 2). We also explored the relationship of each social activity and cognitive impairment in middle-aged and older people at 2-year and 4-year follow-up (see online supplemental appendix 1).

\section{Single and combined effects of different types of social engagement on cognitive impairment}

Table 3 displays the single effects of each type of social engagement on cognitive impairment. At 2-year follow-up, every increase in leisure activities score reduced the likelihood of cognitive impairment by $5 \%$ (OR= $0.95,95 \%$ CI 0.92 to 0.99 , $\mathrm{p}=0.015)$. The results showed a similar pattern in longerterm 4-year follow-up. Meanwhile, at 4-year follow-up, every increase in learning activities score reduced the likelihood of cognitive impairment by $47 \%$ ( $\mathrm{OR}=0.53,95 \%$ CI 0.36 to $0.80, \mathrm{p}=0.002$ ) (table 3). Table 4 demonstrats the second pair of multivariate logistic regression on the combined effect of different types of social engagement on cognitive impairment. At 2-year follow-up, compared with those who did not participate in any social engagement, participants who attended one type of the social engagement (any one of the leisure activities, social activities or learning activities) had a significantly lower risk of developing cognitive impairment $(\mathrm{OR}=0.86,95 \%$ CI 0.76 to $0.96, \mathrm{p}=0.028)$. The protection effect is even larger with the participants who attended two or three types of social engagement $(\mathrm{OR}=0.56,95 \%$ CI 0.41 to $0.76, \mathrm{p}<0.001)$. The results showed a similar pattern in the longer-term 4-year follow-up. Participants who attended one type of social engagement had a $13 \%(\mathrm{OR}=0.87,95 \%$ CI 0.78 to $0.97, \mathrm{p}=0.034$ ) lower risk of developing cognitive impairment, and for those who attended two or three types 
Table 2 Associations of social engagement with incident cognitive impairment in middle-aged and older people at 2-year and 4-year follow-up: The China Health and Retirement Longitudinal Study

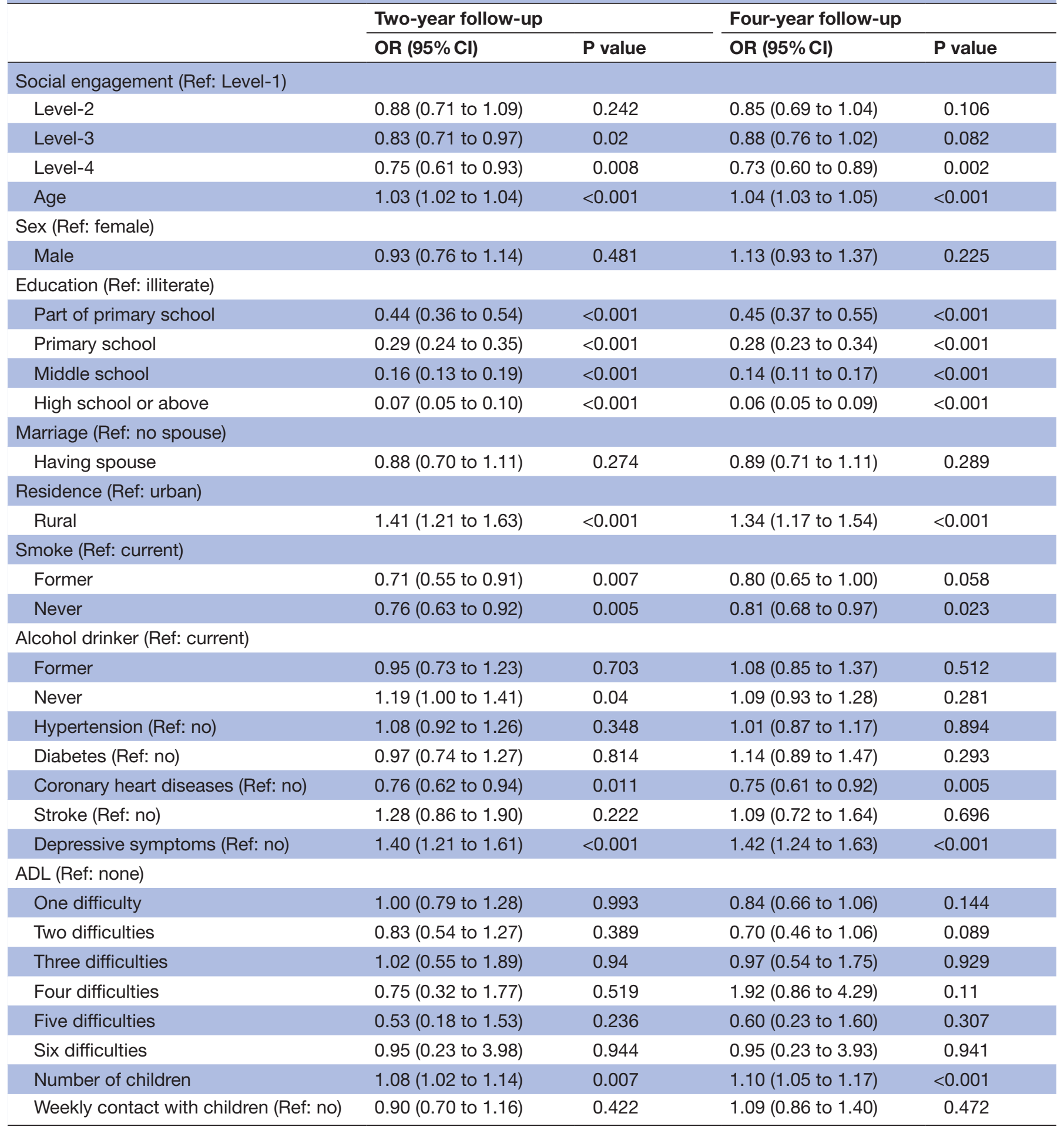

$A D L$, activity of daily living.

of social engagement had a $41 \%(\mathrm{OR}=0.58,95 \% \mathrm{CI} 0.44$ to $0.77, \mathrm{p}<0.001)$ lower risk of developing cognitive impairment (table 4).

\section{DISCUSSIONS}

To our knowledge, this is the first nationally representative study that investigated the prospective association between multidimensional social engagement and the incidence of cognitive impairment in LMICs. This study found that social engagement had a significant protective 
Table 3 The relationship of each type of social engagement and cognitive impairment in middle-aged and older people at 2year and 4-year follow-up: The China Health and Retirement Longitudinal Study*

\begin{tabular}{|c|c|c|c|c|}
\hline & \multicolumn{2}{|l|}{ Two-year follow-up } & \multicolumn{2}{|c|}{ Four-year follow-up } \\
\hline & OR $(95 \% \mathrm{Cl})$ & $P$ value & OR $(95 \% \mathrm{Cl})$ & $P$ value \\
\hline Leisure activities score & 0.95 (0.92 to 0.99$)$ & 0.015 & 0.96 (0.92 to 0.99$)$ & 0.012 \\
\hline Learning activities score & $0.77(0.60$ to 1.00$)$ & 0.053 & $0.53(0.36$ to 0.80$)$ & 0.002 \\
\hline
\end{tabular}

*Models were adjusted by the following covariates: age, sex, education, marriage, residence, smoke, alcohol drinker, hypertension, diabetes, coronary heart diseases, stroke, depressive symptoms, ADL, number of children and weekly contact with children.

ADL, activity of daily living.

effect on cognitive impairment in individuals aged 45 years and over. China has the largest population aged 65 years and older in the world; and in 2050, the proportion of older people is projected to be about $25 \%$. The health needs for older people with irreversible cognitive impairment challenge the health and social care system in a serious and unprecedented way in China and other LMICs. The findings highlighted the need to increase social engagement of middle-aged and older population for delaying the process of cognitive decline and improving cognitive functions in LMICs especially those are stepping into an ageing society.

We found that people with less social engagement were more likely to develop cognitive impairment, which was consistent with previous studies. ${ }^{16}{ }^{16}$ Prior studies pointed out that loneliness, anxiety and poor life satisfaction were all associated with cognitive impairment. ${ }^{32} 33$ On one hand, social engagement provides a platform for middle-aged and older people to communicate with each other, which is helpful for alleviating negative emotions and preventing social isolation. ${ }^{34} 35$ On the other hand, people who attended social activities had more opportunities to stimulating and expanding their minds through meaningful experience. ${ }^{36}$ Just as Berkman described the process as 'use it or lose it', more use of brain may slow down the process of cognitive impairment. In addition, our results confirmed the temporal relationship that social engagement leads to better cognitive function. ${ }^{37}$

We also found a greater protective association between multiple types of social engagement and cognitive impairment relative to single type of social engagement. Different dimensions of activities may have different functions and areas of cognition-related application. Leisure activities, such as playing Mahjong or cards, made people more relaxed and freer from stress. Social activities helping others could provide a sense of fulfilment and companionship, which in turn bolster cognitive functioning. ${ }^{36} 38$ For learning activities, studies showed that internet use provided important platforms for cognitively stimulating activities to delay cognitive decline. ${ }^{24}$ Learning activities may generate a cognitive reserve buffer that promotes resilience to prevent or delay the development of cognitive impairment. ${ }^{39}$ Our results underscored the importance of attending different types of social engagement for delaying the process of cognitive impairment.

In summary, by enhancing social engagement with multiple types of activities, it could effectively alleviate the social and economic burden of cognitive impairment and further dementia among the middle-aged and older people. It is necessary to not only focus on the quantity of type of activities, but also the frequency that individuals take the activities. Generally, to improve social engagement of middle-aged and old population, a national strategy is required with joint actions from different perspectives in a combined effort. The government should create a better social environment by providing platforms for learning advanced knowledge and technologies, encouraging the older adults to actively participate in exercise activities, and increasing the internet penetration rate of older adults. The older-related associations or societies should initiate more social programmes that the older people can benefit from participating in such

Table 4 Combined effects of different types of social engagement on the incidence of cognitive impairment in middle-aged and older people at 2-year and 4-year follow-up: The China Health and Retirement Longitudinal Study*

\begin{tabular}{|c|c|c|c|c|}
\hline & \multicolumn{2}{|l|}{ Two-year follow-up } & \multicolumn{2}{|l|}{ Four-year follow-up } \\
\hline & OR $(95 \% \mathrm{Cl})$ & $P$ value & OR $(95 \% \mathrm{Cl})$ & $P$ value \\
\hline \multicolumn{5}{|c|}{ Social engagement (Ref: none) } \\
\hline Two or three types & $0.56(0.41$ to 0.76$)$ & $<0.001$ & $0.58(0.44$ to 0.77$)$ & $<0.001$ \\
\hline
\end{tabular}

*Models were adjusted by the following covariates: age, sex, education, marriage, residence, smoke, alcohol drinker, hypertension, diabetes, coronary heart diseases, stroke, depressive symptoms, ADL, number of children and weekly contact with children.

ADL, activity of daily living. 
kind of activities. The programmes need to be tailored to local context prior to implementing interventions, so the community can create convenient conditions for various social activities for the older. For families, it is necessary to encourage middle-aged and older people to spend more time on participating in more social activities. In China, intergenerational coresidence rates are relatively high and older adults undertake the responsibility of providing support for their adult children, such as raising their grandchildren and helping with housework, which occupied most of their daily time. ${ }^{15}$ Therefore, it is necessary for family members to give middle-aged and older people more free time to participate in social engagement.

This study has several limitations. First, the observational nature of our study limited our ability to confirm a causal relationship between social engagement and cognitive impairment. Our longitudinal study design facilitates the prospective associations in the present study, which underscored the need for research to capitalise on diverse social engagement to reduce the incident cognitive impairment. Second, due to data feasibility, the quality of social engagement cannot be measured. This might limit our ability to interpret how social engagement work against cognitive impairment. Nevertheless, our study built a comprehensive indicator to measure social engagement, taking into account the quantity and frequency of social engagement. Third, cognitive impairment measures were based on subjective assessment rather clinical diagnosis, which may modify the estimated associations between social engagement and cognitive impairment. Despite these limitations, the strengths of this study included a national representative and population-based sample with 4 years of follow-up, the extracted participants who had cognitive impairment at baseline to identify the effect of the social engagement, as well as multidimensional constructs of social engagement and cognitive impairment.

\section{CONCLUSION}

Social engagement had a protective effect on cognitive impairment in middle-aged and older adults in China. Different types of activities should be encouraged to delay cognitive decline at mid-age and later life. Future studies are warranted to explore the causal relationship and multiple mechanisms between social engagement and cognitive impairment. Given the growing epidemic of cognitive impairment and rapid pace of ageing in LMICs, we believed that our study shed lights on the actions to developing comprehensive and tailored programmes for improving the enrolment of social engagement, to slow down the speed of cognitive impairment among the middle-aged and older population.

Acknowledgements We would like to thank all coauthors. We would also like to extend our thanks to the China Health and Retirement Longitudinal Study team for providing data and the training of using the dataset.

Contributors SZ: drafting the manuscript, study design, data analysis and interpretation. SS: critical revision of article for important intellectual content. YJ: study concept and design, data analysis and critical revision of article for important intellectual content. Z-JZ: critical revision of article for important intellectual content. All authors gave final approval of the version to be published.

Funding This paper was supported by the Beijing Municipal Natural Science Foundation (No. 9204025). The study sponsor has no role in study design, data analysis and interpretation of data, the writing of manuscript, or the decision to submit the paper for publication.

Competing interests None declared.

Patient consent for publication Not required.

Ethics approval All participants provided written informed consent, and ethical approval for collecting data on human subjects was received and updated annually at Peking University Institutional Review Board (IRB00001052-11015). Ethics approval for the use of China Health and Retirement Longitudinal Study data was obtained from the University of Newcastle Human Research Ethics Committee (H-2015-0290).

Provenance and peer review Not commissioned; externally peer reviewed.

Data availability statement Data are available in a public, open access repository at http://charls.pku.edu.cn.

Supplemental material This content has been supplied by the author(s). It has not been vetted by BMJ Publishing Group Limited (BMJ) and may not have been peer-reviewed. Any opinions or recommendations discussed are solely those of the author(s) and are not endorsed by BMJ. BMJ disclaims all liability and responsibility arising from any reliance placed on the content. Where the content includes any translated material, BMJ does not warrant the accuracy and reliability of the translations (including but not limited to local regulations, clinical guidelines, terminology, drug names and drug dosages), and is not responsible for any error and/or omissions arising from translation and adaptation or otherwise.

Open access This is an open access article distributed in accordance with the Creative Commons Attribution Non Commercial (CC BY-NC 4.0) license, which permits others to distribute, remix, adapt, build upon this work non-commercially, and license their derivative works on different terms, provided the original work is properly cited, appropriate credit is given, any changes made indicated, and the use is non-commercial. See: http://creativecommons.org/licenses/by-nc/4.0/.

\section{ORCID iDs}

Shuduo Zhou http://orcid.org/0000-0002-5463-3116

Yinzi Jin http://orcid.org/0000-0003-0634-3955

\section{REFERENCES}

1 Park HL, O'Connell JE, Thomson RG. A systematic review of cognitive decline in the general elderly population. Int J Geriatr Psychiatry 2003;18:1121-34.

2 Roberts RO, Knopman DS, Mielke MM, et al. Higher risk of progression to dementia in mild cognitive impairment cases who revert to normal. Neurology 2014;82:317-25.

3 Prince MJ. World Alzheimer report 2015: the global impact of dementia: an analysis of prevalence, incidence, cost and trends. Alzheimer's Dis Inter 2015.

4 Singh-Manoux A, Kivimaki M, Glymour MM, et al. Timing of onset of cognitive decline: results from Whitehall II prospective cohort study. BMJ 2012;344:d7622.

5 Sliwinski MJ, Hofer SM, Hall C, et al. Modeling memory decline in older adults: the importance of preclinical dementia, attrition, and chronological age. Psychol Aging 2003;18:658-71.

6 Plassman BL, Williams JW, Burke JR, et al. Systematic review: factors associated with risk for and possible prevention of cognitive decline in later life. Ann Intern Med 2010;153:182-93.

7 Zhang Z, Li LW, Xu H, et al. Does widowhood affect cognitive function among Chinese older adults? SSM Popul Health 2019;7:100329.

8 Blazer DG, Yaffe K, Liverman CT. Cognitive aging: progress in understanding and opportunities for action. Washington, DC: National Academies Press, 2015.

9 Zeng Y, Feng Q, Hesketh T, et al. Survival, disabilities in activities of daily living, and physical and cognitive functioning among the oldestold in China: a cohort study. Lancet 2017;389:1619-29.

10 Youssim I, Hank K, Litwin H. The role of family social background and inheritance in later life volunteering: evidence from SHAREIsrael. Res Aging 2015;37:3-17. 
11 Krueger KR, Wilson RS, Kamenetsky JM, et al. Social engagement and cognitive function in old age. Exp Aging Res 2009;35:45-60.

12 Barnes LL, Mendes de Leon CF, Wilson RS, et al. Social resources and cognitive decline in a population of older African Americans and whites. Neurology 2004;63:2322-6.

13 Bassuk SS, Glass TA, Berkman LF. Social disengagement and incident cognitive decline in community-dwelling elderly persons. Ann Intern Med 1999;131:165-73.

14 Stern Y. What is cognitive reserve? Theory and research application of the reserve concept. J Int Neuropsychol Soc 2002;8:448-60.

15 Amano T, Morrow-Howell N, Park S. Patterns of social engagement among older adults with mild cognitive impairment series B. $J$ Gerontol 2019;3:S113.

16 Wang $\mathrm{H}-\mathrm{X}$, Jin $\mathrm{Y}$, Hendrie $\mathrm{HC}$, et al. Late life leisure activities and risk of cognitive decline. J Gerontol A Biol Sci Med Sci 2013;68:205-13.

17 Thomas PA. Trajectories of social engagement and limitations in late life. J Health Soc Behav 2011;52:430-43.

18 Crowe M, Andel R, Pedersen NL, et al. Does participation in leisure activities lead to reduced risk of Alzheimer's disease? A prospective study of Swedish twins. J Gerontol B Psychol Sci Soc Sci 2003;58:P249-55.

19 Murayama H, Fujiwara Y, Kawachi I. Social capital and health: a review of prospective multilevel studies. J Epidemiol 2012;22:179-87.

20 Choi Y, Park S, Cho KH, et al. A change in social activity affect cognitive function in middle-aged and older Koreans: analysis of a Korean longitudinal study on aging (2006-2012). Int J Geriatr Psychiatry 2016;31:912-9.

21 Prince MJ. World Alzheimer Report 2015: the global impact of dementia: an analysis of prevalence, incidence, cost and trends[M] Alzheimer's Dis Inter 2015.

22 Mukadam N, Sommerlad A, Huntley J, et al. Population attributable fractions for risk factors for dementia in low-income and middleincome countries: an analysis using cross-sectional survey data. Lancet Glob Health 2019;7:e596-603.

23 Zhao Y, Hu Y, Smith JP, et al. Cohort profile: the China health and retirement longitudinal study (CHARLS). Int $J$ Epidemiol 2014;43:61-8.

24 Jin $Y$, Jing M, Ma X. Effects of digital device ownership on cognitive decline in a middle-aged and elderly population: longitudinal observational study. J Med Internet Res 2019;21:e14210.

25 Wang T, Wu Y, Sun Y, et al. A prospective study on the association between uric acid and cognitive function among middle-aged and older Chinese. J Alzheimers Dis 2017;58:79-86.
26 Fong TG, Fearing MA, Jones RN, et al. Telephone interview for cognitive status: creating a crosswalk with the Mini-Mental state examination. Alzheimer's \& Dementia 2009;5:492-7.

27 Crimmins EM, Kim JK, Langa KM, et al. Assessment of cognition using surveys and neuropsychological assessment: the health and retirement study and the aging, demographics, and memory study. $J$ Gerontol B Psychol Sci Soc Sci 2011;66:i162-71.

28 Alzheimer's Association. 2010 Alzheimer's disease facts and figures. Alzheimer's \& Dementia 2010;6:158-94.

29 Heine C, Browning CJ, Gong CH. Sensory loss in China: prevalence, use of AIDS, and impacts on social participation. Front Public Health 2019;7:5.

30 World Health Organization, \& World Health Organization Staff. International classification of functioning, disability and health: ICF. World Health Organization, 2001.

31 Bourassa KJ, Memel M, Woolverton C, et al. Social participation predicts cognitive functioning in aging adults over time: comparisons with physical health, depression, and physical activity. Aging Ment Health 2017;21:133-46.

32 Zhong B-L, Chen S-L, Tu X, et al. Loneliness and cognitive function in older adults: findings from the Chinese longitudinal healthy longevity survey. J Gerontol B Psychol Sci Soc Sci 2017;72:120-8.

33 Zhou Z, Wang P, Fang Y. Loneliness and the risk of dementia among older Chinese adults: gender differences. Aging Ment Health 2018;22:519-25.

34 Cohen S. Social relationships and health. Am Psychol 2004;59:676-84.

35 Seeman TE, Lusignolo TM, Albert M, et al. Social relationships, social support, and patterns of cognitive aging in healthy, high-functioning older adults: MacArthur studies of successful aging. Health Psychol 2001;20:243-55

$36 \mathrm{Pan} \mathrm{X}$, Chee $\mathrm{KH}$. The power of weak ties in preserving cognitive function: a longitudinal study of older Chinese adults. Aging Mental Health 2019:1-8.

37 Lee Y, Jean Yeung W-J, Yeung WJJ. Gender matters: productive social engagement and the subsequent cognitive changes among older adults. Soc Sci Med 2019;229:87-95

38 Tu R, Inoue Y, Yazawa A, et al. Social participation and the onset of hypertension among the middle-aged and older population: evidence from the China health and retirement longitudinal study. Geriatr Gerontol Int 2018;18:1093-9.

39 Livingston G, Sommerlad A, Orgeta V, et al. Dementia prevention, intervention, and care. Lancet 2017;390:2673-734. 